\title{
DIAGRAMS OF STATISTICAL STRENGTH CRITERION FOR REINFORCED COMPOSITE MATERIALS
}

\author{
Roman Kvit, Tetyana Salo \\ Lviv Polytechnic National University \\ Lviv, Ukraine \\ kvit_rom@ukr.net,tetyan_salo@gmail.com
}

Received: 21 February 2021; Accepted: 1 June 2021

\begin{abstract}
The model of a plate brittle material reinforced with randomly sized and placed rigid rectilinear inclusions that do not interact with each other is considered. The geometric parameters of inclusions (length and orientation) are statistically independent random variables, with certain given laws of probability distribution. Diagrams of statistical strength criterion for such plates are constructed under conditions of comprehensive tension-compression. The diagrams are constructed for plates using a material of a different structural inhomogeneity and Poisson's ratio of an elastic homogeneous matrix. The statistical nature of the scale effect is studied, the intensity of which depends on the type of stress state.
\end{abstract}

MSC 2010: 74R10, 74R99, 60K35, 82C03

Keywords: composite material, rigid inclusion, distribution function, strength criterion, failure loading

\section{Introduction}

The study of the strength and reliability of construction elements with structural inhomogeneity of materials, which may be caused by extraneous inclusions, is an urgent task in the mechanics of composite materials. An important step in studying the stress state and the failure of construction elements under a given load is their probabilistic estimation. Defects in the structure of a material, and therefore its strength, are always random variables. Therefore, the use of probability-statistical methods is a prerequisite for calculating the reliability of materials. The complex application of these methods and the well-known deterministic solutions of material fracture mechanics make it possible to obtain more accurate models of the strength criteria of machine parts and construction elements under different loading conditions. The application of probabilistic-statistical methods in predicting the reliability of materials with structural inhomogeneity has been developed in the works of a number of authors. In paper [1], the reliability calculation of composite material using a variety of approximation methods is described and illustrated. Article [2] 
presents a methodology for reliability assessment of composite members based on appropriate limit state functions derived according to fundamental failure criteria, applicable to composite materials. A two-dimensional finite element simulation-based approach was developed [3] to assess the fracture statistics of isotropic microstructures. An analytical approach with the help of numerical simulations based on the equivalent constraint model was proposed [4] to investigate the progressive failure behavior of symmetric fiber-reinforced composite laminates damaged by transverse ply cracking. A modified Weibull failure probability model that considers the impact of compressive stress on cladding failure probability is deduced in detail [5]. In study [6], reliability analysis of tensile strengths using Weibull distribution in glass/epoxy and carbon/epoxy composites is carried out. In article [7], the expressions for the probabilities density distribution of failure loading for an elastic isotropic body under the action of a homogeneous axisymmetric loading are written. The correlation for finding the most probable, mean value, dispersion and variation coefficient of failure loading is obtained. In this work, the application of an integrated approach based on the provisions of fracture mechanics of materials and methods of the theory of probability to reinforced composite materials is continued.

The purpose of this study is to construct and analyze diagrams of the statistical criterion for the strength of a brittle material plate, in which stochastically random rigid linear (rod) inclusions are distributed under different loading conditions.

\section{Formulation of the problem}

Consider a brittle rectangular plate under conditions of comprehensive tensilecompression in two mutually perpendicular directions with uniform loading $P$ and $Q=\eta P$ (Fig. 1). Rigid rod $N$ inclusions that do not interact with each other are evenly distributed in it. The values $P$ and $Q$ will be considered as the main stresses for a plane stress state. The inclusions are characterized by the length $2 l$ $(0 \leq 2 l \leq c, c$ is a finite structural characteristic) and the angle $\alpha$ of their inclination $(-\pi / 2 \leq \alpha \leq \pi / 2)$ relative to the direction of force action $P$. The parameters $\alpha$ and $l$ are statistically independent random variables.

To describe the random variable $l$, we use the beta distribution [8], the density of which

$$
f(l)=\frac{r+1}{c}(1-l / c)^{r} .
$$

It is an important statistical model for random variables whose values are limited by a finite interval. The parameter $r \geq 0$ characterizes the structure of the material. With $r=0$, the beta distribution turns into a uniform distribution. For $r=1$, a linearly decreasing distribution is obtained. In the case $r>0$, with an increase in the parameter $r$, the probability of encountering random variables close to zero is 
greater, and the lower the probability of encountering large values of a random variable. Therefore, by increasing $r$, the inclusions of small size are more likely.

Then the integral distribution function of a random variable $l$ can be written as

$$
F(l)=1-(1-l / c)^{r+1} .
$$

To describe the random variable $\alpha$, we use the density of a uniform probability distribution

$$
f(\alpha)=1 / \pi
$$

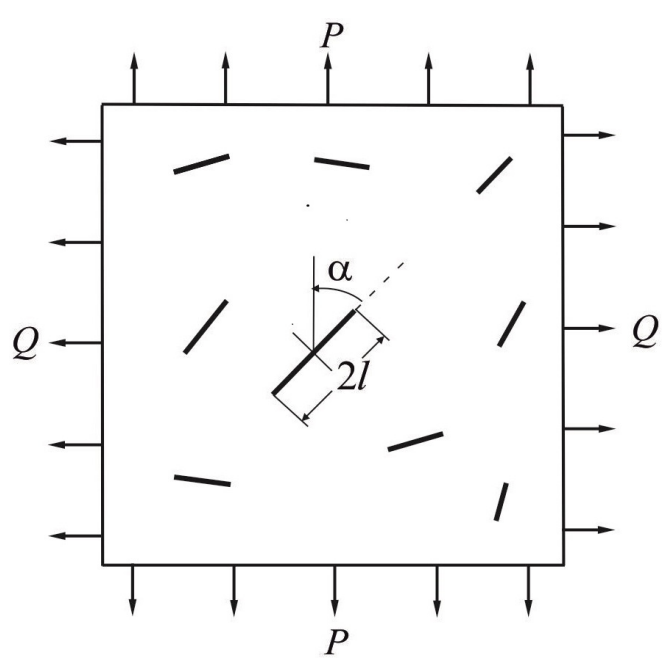

Fig. 1. Plate with inclusions under conditions of comprehensive tensile-compression

In article [9], the expressions of the failure loading distribution function $F_{1}(P, \eta)$ of a plate element with one inclusion of the specified type were obtained for different ratios of the applied loading. These expressions were obtained using the integral distribution function of a random variable $l(2)$, the uniform distribution density of the random variable $\alpha$ (3), and deterministic fracture conditions in the vicinity of the inclusion [10]. Substitute the expressions $F_{1}(P, \eta)$ into the equation of the statistical strength diagram for the plate with $N$ inclusions, which is recorded in the mean values of the failure loading [10]

$$
\langle P\rangle=P_{\min }(\eta)+\int_{P_{\min }(\eta)}^{P_{\max }(\eta)}\left(1-F_{1}(P, \eta)\right)^{N} d P,\langle Q\rangle=\eta\langle P\rangle .
$$

This strength diagram depends on the distribution law of the size of inclusions (parameter $r$ ), the nature of the stress state (parameter $\eta$ ), plate dimensions (we consider the number of inclusions $N$ proportional to the plate area) and the Poisson's ratio $v$ of the plate material. 
We replace the variables $x=\frac{K_{0}^{2}}{P^{2} c}$ (the constant $K_{0}$ characterizes the resistance of the material to local failure [10]). We obtain the following expressions for the mean value of the failure loading (we write it as a dimensionless value $\langle P\rangle \sqrt{c} / K_{0}$ ) for different types of applied loading:

1) for the case $\frac{3-æ}{1+\mathfrak{x}} \leq \eta \leq 1(P>0)$

$$
\begin{aligned}
& \langle P\rangle \frac{\sqrt{c}}{K_{0}}=\frac{1}{\chi_{1}(\eta, \mathfrak{x})+\chi_{2}(\eta, \mathfrak{x})}+\frac{1}{2} \int_{0}^{\left(\chi_{1}(\eta, \mathfrak{x})-\chi_{2}(\eta, \mathfrak{x})\right)^{2}} \frac{\left(1-\Psi_{2}(x, \eta, \mathfrak{x})\right)^{N}}{x^{3 / 2}} d x+ \\
& +\frac{1}{2} \int_{\left(\chi_{1}(\eta, \mathfrak{x})-\chi_{2}(\eta, \mathfrak{x})\right)^{2}}^{\left(\chi_{1}(\eta, \mathfrak{x})+\chi_{2}(\eta, \mathfrak{x})\right)^{2}} \frac{\left(1-\Psi_{1}(x, \eta, \mathfrak{x})\right)^{N}}{x^{3 / 2}} d x ;
\end{aligned}
$$

2) for the case $-1 \leq \eta \leq \frac{3-\mathfrak{x}}{1+\mathfrak{x}}(P>0)$

$$
\begin{aligned}
& \langle P\rangle \frac{\sqrt{c}}{K_{0}}=\frac{1}{\chi_{1}(\eta, \mathfrak{x})+\chi_{2}(\eta, \mathfrak{x})}+\frac{1}{2} \int_{0}^{\left(\chi_{1}(\eta, \mathfrak{x})+\chi_{2}(\eta, \mathfrak{x}) \cos 2 \alpha_{1}\right)^{2}} \frac{\left(1-\Psi_{3}(x, \eta, \mathfrak{x})\right)^{N}}{x^{3 / 2}} d x+ \\
& +\frac{1}{2} \int_{\left(\chi_{1}(\eta, \mathfrak{x})+\chi_{2}(\eta, \mathfrak{x}) \cos 2 \alpha_{1}\right)^{2}} \frac{\left(1-\Psi_{1}(x, \eta, \mathfrak{x})\right)^{N}}{x^{3 / 2}} d x ;
\end{aligned}
$$

3) for the case $-\infty<\eta<-1(P>0)$

$$
\begin{aligned}
& \langle P\rangle \frac{\sqrt{c}}{K_{0}}=\frac{1}{\chi_{1}(\eta, \mathfrak{x})+\chi_{2}(\eta, \mathfrak{x})}+\frac{1}{2} \int_{0}^{\left(\chi_{3}(\eta, \mathfrak{x})+\chi_{4}(\eta, \mathfrak{x}) \cos 2 \alpha_{1}\right)^{2}} \frac{\left(1-\Psi_{4}(x, \eta, \mathfrak{x})\right)^{N}}{x^{3 / 2}} d x+ \\
& +\frac{1}{2} \int_{\left(\chi_{3}(\eta, \mathfrak{x})+\chi_{4}(\eta, \mathfrak{x}) \cos 2 \alpha_{1}\right)^{2}} \frac{\left(1-\Psi_{3}(x, \eta, \mathfrak{x})\right)^{N}}{x^{3 / 2}} d x ;
\end{aligned}
$$

4) for the case $1 \leq \eta \leq \frac{1+\mathfrak{x}}{3-\mathfrak{x}}(P<0)$

$$
\begin{aligned}
& \langle P\rangle \frac{\sqrt{c}}{K_{0}}=-\frac{1}{\chi_{3}(\eta, \mathfrak{x})+\chi_{4}(\eta, \mathfrak{x})}-\frac{1}{2} \int_{0}^{\left(\chi_{3}(\eta, \mathfrak{x})+\chi_{4}(\eta, \mathfrak{x})\right)^{2}} \frac{\left(1-\Psi_{6}(x, \eta, \mathfrak{x})\right)^{N}}{x^{3 / 2}} d x- \\
& -\frac{1}{2} \int_{\left(\chi_{3}(\eta, \mathfrak{x})+\chi_{4}(\eta, \mathfrak{x})\right)^{2}}^{\left(\chi_{3}(\eta, \mathfrak{x})-\chi_{4}(\eta, \mathfrak{x})\right)^{2}} \frac{\left(1-\Psi_{5}(x, \eta, \mathfrak{x})\right)^{N}}{x^{3 / 2}} d x ;
\end{aligned}
$$


5) for the case $\frac{1+\mathfrak{x}}{3-\mathfrak{x}} \leq \eta<\infty(P<0)$

$$
\begin{aligned}
& \langle P\rangle \frac{\sqrt{c}}{K_{0}}=-\frac{1}{\chi_{3}(\eta, \mathfrak{x})+\chi_{4}(\eta, \mathfrak{x}) \cos 2 \alpha_{1}}-\frac{1}{2} \int_{0}^{\left(\chi_{3}(\eta, \mathfrak{x})+\chi_{4}(\eta, \mathfrak{x}) \cos 2 \alpha_{1}\right)^{2}} \frac{\left(1-\Psi_{3}(x, \eta, \mathfrak{x})\right)^{N}}{x^{3 / 2}} d x- \\
& -\frac{1}{2} \int_{\left(\chi_{3}(\eta, \mathfrak{x})+\chi_{4}(\eta, \mathfrak{x})\right)^{2}}^{\left(\chi_{3}(\eta, \mathfrak{x})-\chi_{4}(\eta, \mathfrak{x}) \cos 2 \alpha_{1}\right)^{2}} \frac{\left(1-\Psi_{5}(x, \eta, \mathfrak{x})\right)^{N}}{x^{3 / 2}} d x
\end{aligned}
$$

The following notation is used here:

$$
\begin{aligned}
& \Psi_{1}(x, \eta, æ)=\frac{2}{\pi} \int_{0}^{\alpha_{2}}\left(1-x \varphi_{1}^{2}(\alpha, \eta, æ)\right)^{r+1} d \alpha ;
\end{aligned}
$$

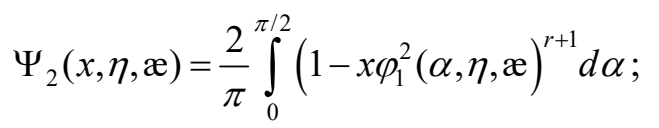

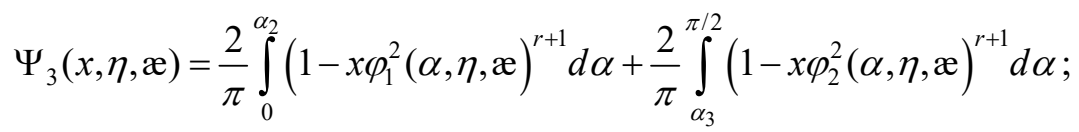

$$
\begin{aligned}
& \Psi_{4}(x, \eta, æ)=\frac{2}{\pi} \int_{0}^{\alpha_{1}}\left(1-x \varphi_{1}^{2}(\alpha, \eta, æ)^{r+1} d \alpha+\frac{2}{\pi} \int_{\alpha_{1}}^{\pi / 2}\left(1-x \varphi_{2}^{2}(\alpha, \eta, æ)^{r+1} d \alpha\right.\right. \\
& \Psi_{5}(x, \eta, æ)=\frac{2}{\pi} \int_{\alpha_{3}}^{\pi / 2}\left(1-x \varphi_{2}^{2}(\alpha, \eta, æ)^{r+1} d \alpha\right.
\end{aligned}
$$

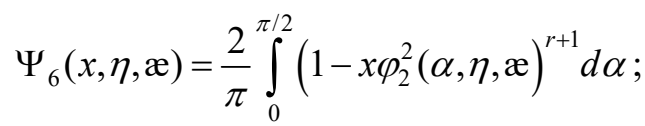

$$
\begin{aligned}
& \varphi_{1}(\alpha, \eta, æ)=\left(\chi_{1}(\eta, æ)+\chi_{2}(\eta, æ) \cos 2 \alpha\right)^{-1} ; \\
& \varphi_{2}(\alpha, \eta, æ)=\left(\chi_{3}(\eta, æ)+\chi_{4}(\eta, æ) \cos 2 \alpha\right)^{-1} ;
\end{aligned}
$$

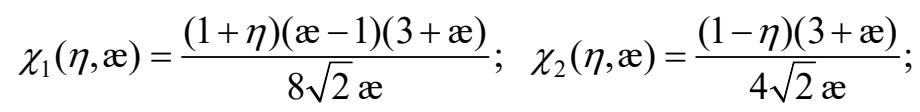

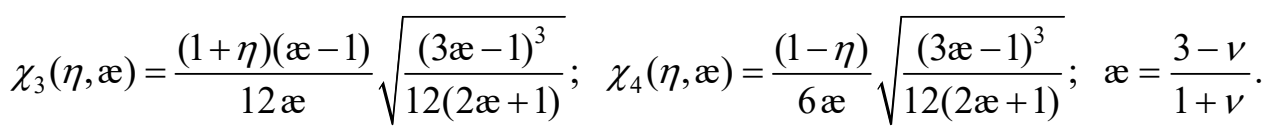


The limits of integration are defined as follows:

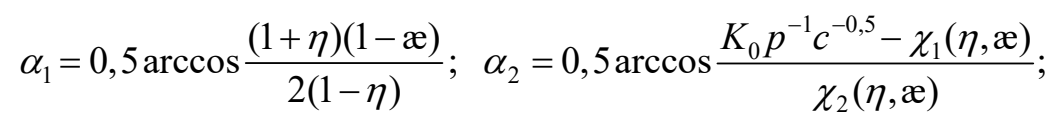

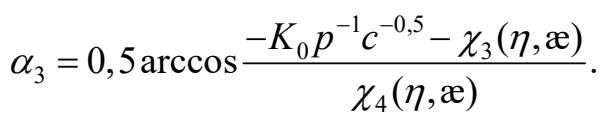

We find the dimensionless mean value of the failure loading second component $\langle Q\rangle \sqrt{c} / K_{0}$ according to expression (4).

In article [9], the following partial cases of loading of a plate with $N$ rigid rod inclusions are considered: uniaxial tension $(\eta=0)$, biaxial symmetric tension $(\eta=1)$ and biaxial symmetric tension-compression $(\eta=-1)$.

The dependences of the failure loading mean value $\langle P\rangle$ on the parameters $r$ and $N$ on the fixed $\eta$ and $æ$ will be similar to those of plates with defects-cracks $[10,11]$. The dependence $\langle P\rangle$ on the $\eta$ will be different not only because of the influence of the parameter $r$, but also because of the influence of the Poisson's ratio $v$ of the matrix. Let's show this in more detail by comparing a two-axis symmetric tension with a uniaxial tension.

Introduce the notation $\lambda=\left.\langle P\rangle\right|_{\eta=1} /\left\langle\left. P\right|_{\eta=0}\right.$ and make the appropriate calculations. Figure 2 shows the dependence of the value $\lambda$ on the parameter $r$ and the Poisson's ratio $v$ (at a fixed value $N=50$ ). For plates with cracks, the curves of such dependencies with increasing parameter $r$ are asymptotically directed to one [10], which corresponds to the case of a completely homogeneous material. This pattern for the plates with inclusions is not traceable. With increasing $r$ values $\lambda$ go to some boundary that depends on $v$.

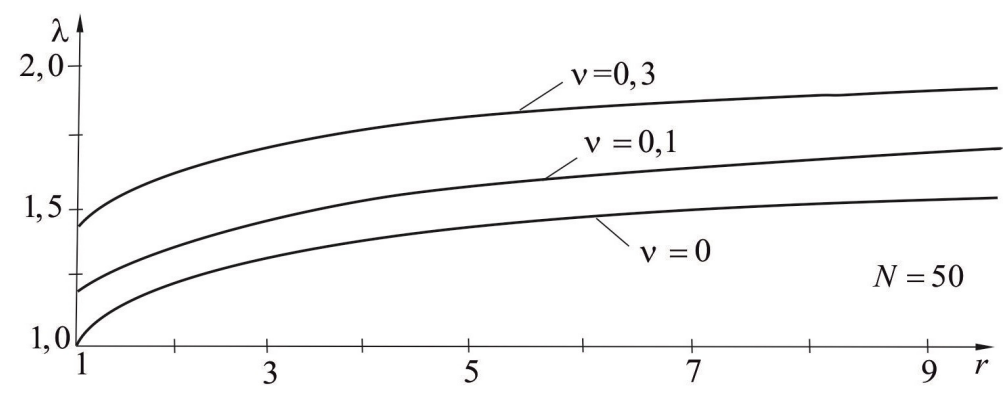

Fig. 2. Dependence of parameter $\lambda$ on material characteristics $r$ and $v$ 


\section{Statistical diagrams of composite materials}

Consider constructing statistical strength diagrams of plates with stochastically distributed rigid rod inclusions recorded in the mean values of the failure loading in accordance with expressions (4)-(9).

In Figure 3, the diagrams for the case of choosing the probability density of a random variable $l$ distribution in the form of a linearly decreasing law $f(l)=\frac{2}{c}(1-l / c)$ (according to the expression (1)) are considered. This distribution probability density reflects the following property: the longer the inclusion, the less likely it will occur in the material. Strength diagrams are constructed for plates with a different number $N$ of inclusions and different values of the Poisson's ratio $v$ of the plate material.

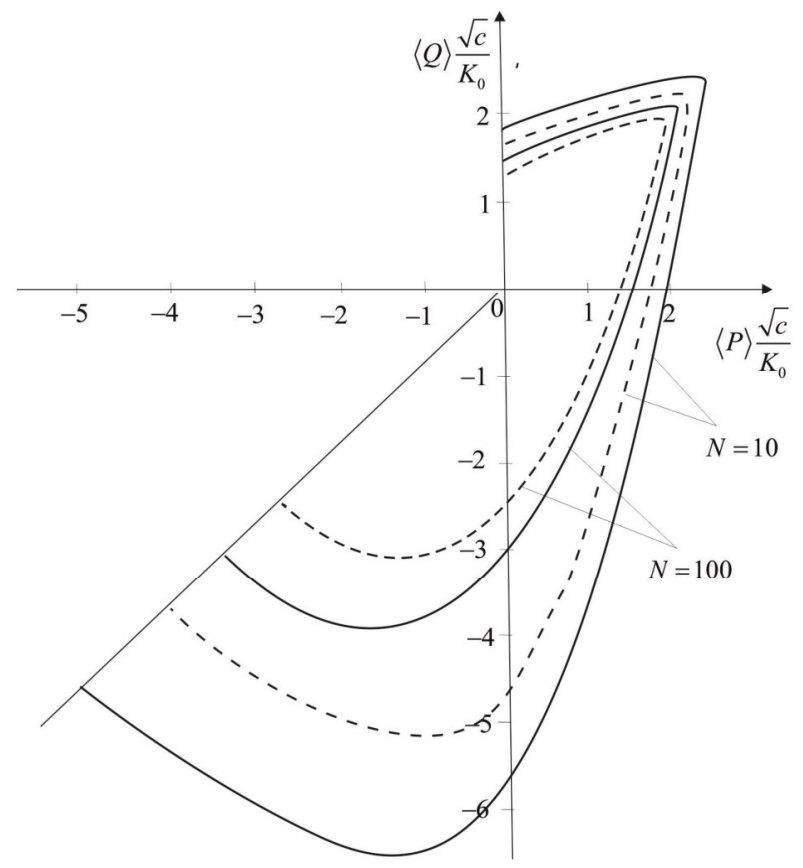

Fig. 3. Strength diagrams for different number of inclusions (solid lines for $v=0.3$, dashed lines for $v=0.1$ )

Figure 4 shows the statistical strength diagrams of plates with fixed sizes $(N=100)$ and different structural inhomogeneity of the material (parameter $r$ ).

According to the choice of the uniform probability distribution density of the random variable $\alpha$ (3), the constructed strength diagrams are symmetric about the straight line $\langle Q\rangle=\langle P\rangle$. 


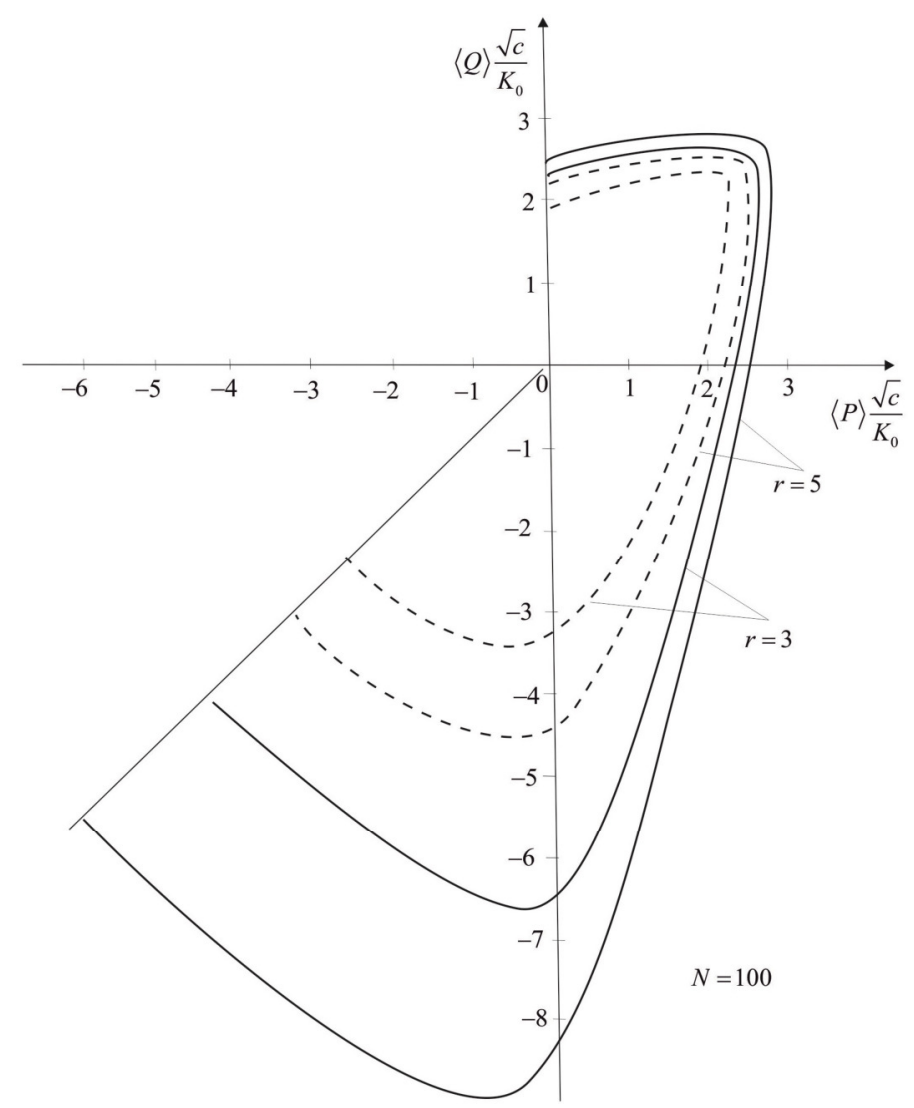

Fig. 4. Strength diagrams for different material structural inhomogeneity (solid lines for $v=0.3$, dashed lines for $v=0.1$ )

\section{Conclusions}

The strength diagrams for different inclusions (Fig. 3) indicate the scale effect of the plates strength. As the number of inclusions $N$ (the size of the plate) increases, a scale effect that is statistical in nature is manifested. The intensity of the scale effect does not depend on the type of stress state, as evidenced by the similarity of curves of mean strength, which correspond to the same parameters $v$ and $r$ at a different number of inclusions (at different plate sizes). With a decrease in the Poisson's ratio of the plate material, the mean value of the failure loading decreases. The magnitude of the reduction in the failure loading depends on the type of stress state and is significant in the region of prevailing compression. Figure 4 shows the effect of changes in the structural inhomogeneity of the material on the mean value of the failure loading. The strength of the specimen increases with increasing of value $r$ at constant plate sizes. This pattern can be explained by the increase in the uniformity of the material (the physical content of the parameter $r$ ). Also, 
the strength of the composite is affected by the complication of the stress state. The effect of the Poisson's ratio on the change in the shape of the mean strength curves is negligible.

\section{References}

[1] Wetherhold, R., \& Ucci, A. (1994). Probability methods for the fracture of composite materials. Composite Structures, 28(15), 113-119.

[2] Kolios, J., \& Proia, S. (2012). Evaluation of the reliability performance of failure criteria for composite structures. World Journal of Mechanics, 2, 162-170.

[3] Keles, Ö., Garcia, R., \& Bowman, K. (2013). Stochastic failure of isotropic brittle materials with uniform porosity. Acta Materialia, 61(8), 2853-2862.

[4] Wang, F., Ding, J., \& Chen, Z. (2014). Statistical analysis of the progressive failure behavior for fiber-reinforced polymer composites under tensile loading. Polymers, 6, 145-159.

[5] Zhang, T., Yue, R., Wang, X., \& Hao, Z. (2018). Failure probability analysis and design comparison of multi-layered sic-based fuel cladding in PWRs. Nuclear Engineering and Design, 330, 463-479.

[6] Naresh, K., Shankar, K., \& Velmurugan, R. (2018). Reliability analysis of tensile strengths using Weibull distribution in glass/epoxy and carbon/epoxy composites. Composites Part B: Engineering, 133(15), 129-144.

[7] Kvit, R. (2018). Strength statistical characteristics of the isotropic materials with disk-shaped defects. Journal of Applied Mathematics and Computational Mechanics, 17(4), 25-34.

[8] Kvit, R., \& Salo, T. (2011). A probabilistic design of composite materials reliability. Mechanical Engineering, 9-10, 21-27 (in Ukrainian).

[9] Kvit, R. (2011). About statistical strength characteristics of composite materials. Visnyk of the Lviv University. Series Mechanics and Mathematics, 75, 127-136 (in Ukrainian).

[10] Vytvytsky, P., \& Popina, S. (1980). Strength and Criteria of Brittle Fracture of Stochastically Defective Bodies. Kyiv, 186 (in Russian).

[11] Kvit, R. (2018). Construction of the strength statistical criteria with consideration of some brittle materials failure deterministic aspects features. Precarpathian Bulletin of the Shevchenko Scientific Society, 2(46), 119-127 (in Ukrainian). 